\title{
Rectal varices in extrahepatic portal vein obstruction
}

\author{
Abhirup Banerjee $^{1}$ - Sudeep R. Shah ${ }^{1}$ - Philip Abraham ${ }^{2}$
}

Published online: 27 May 2015

(C) Indian Society of Gastroenterology 2015

Varices located outside the esophagogastric junction (ectopic varices) can be located in several sites. Rectal varices occur in $44 \%$ to $78 \%$ of patients with portal hypertension and are significantly more common in patients with noncirrhotic portal hypertension compared to patients with cirrhosis ( $89 \%$ vs. $56 \%$ ) [1]. Although they are potentially a serious cause of lower gastrointestinal (GI) bleeding, massive bleeding is rare. These varices represent dilated portosystemic shunting between the superior rectal veins (inferior mesenteric system) and the middle/inferior rectal veins (internal iliac system). It is important to differentiate them from hemorrhoids; a misdiagnosis may have disastrous consequences in management. Unlike hemorrhoids, rectal varices extend beyond $4 \mathrm{~cm}$ from the anal verge, collapse with digital pressure, and do not prolapse into the proctoscope. Transjugular intrahepatic portosystemic shunt (TIPS) placement, endoscopic band ligation, endoscopic injection sclerotherapy, and underrunning the varix with suture are the main treatment options available [2].

The accompanying image is from a patient with extrahepatic portal vein obstruction, who had undergone a proximal splenorenal shunt 12 years ago and presented now with lower GI bleeding due to shunt occlusion. The rectal varices (Fig. 1) were successfully treated with endoscopic injection of cyanoacrylate glue.

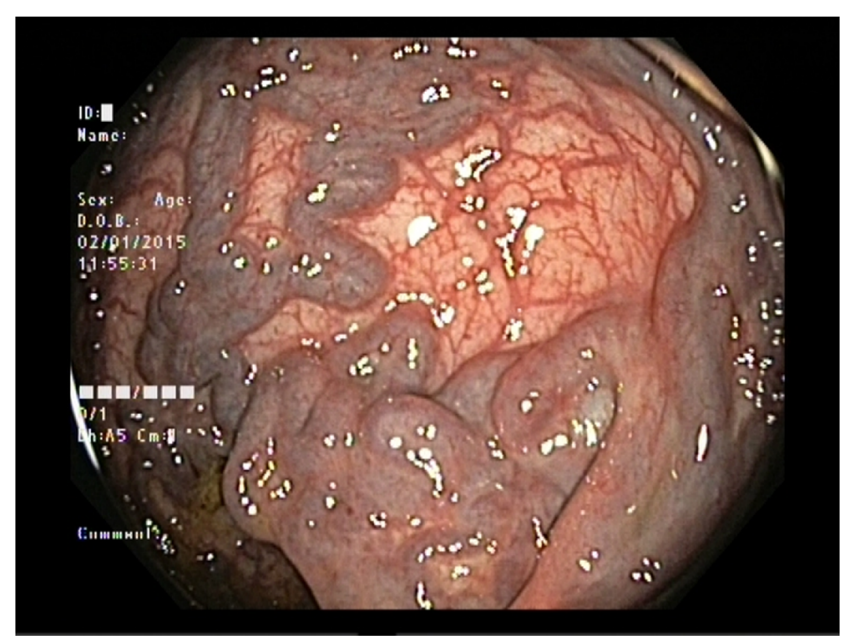

Fig. 1 Rectal varices seen during colonoscopy

\section{References}

1. Chawla Y, Dilawari JB. Anorectal varices - their frequency in cirrhotic and non-cirrhotic portal hypertension. Gut. 1991;32:309-11.

2. Sato T. Treatments for rectal varices with portal hypertension. J Gastroint Dig Syst. 2013;S6:005.
Abhirup Banerjee

drabhirupbanerjee@gmail.com

1 Division of Gastrointestinal Surgery, P D Hinduja National Hospital and Medical Research Centre, Veer Savarkar Marg, Mahim, Mumbai 400 016, India

2 Department of Gastroenterology, P D Hinduja National Hospital and Medical Research Centre, Veer Savarkar Marg, Mahim, Mumbai 400 016, India 